\title{
The Feasibility of Measuring Turbulence in the Free Atmosphere from Rising Balloons Tracked by FPS-16 Radar ${ }^{1}$
}

\author{
R. M. Enduich and J. W. Davies \\ Stanford Research Institute, Menlo Park, Calif. \\ (Manuscript received 27 June 1966, in revised form 21 August 1966)
}

\section{ABSTRACT}

\begin{abstract}
On passing through an atmospheric layer that contains random isotropic turbulent eddies, one would expect that a rising balloon would follow an erratic path. Recently, an FPS-16 missile-tracking radar has been used to follow the paths of special balloons that have only small aerodynamic oscillatory motions. To detect turbulent motions, the slant-range measurements are smoothed, and deviations from the smoothed curve are computed and plotted. Ten flights of "Jimsphere" balloons have been processed in this manner. The slant-range deviations reveal small regular self-induced motions and occasional turbulent layers. Observations of this type provide a means of measuring turbulence from low levels into the stratosphere, and also give concurrent wind measurements. It appears that correlations between properties of the turbulence and the associated wind profile can be determined with greater confidence from these data than from any other turbulence measurements presently available.
\end{abstract}

\section{Introduction}

In spite of the attention directed toward free air turbulence in recent years, measurements of this atmospheric state are not obtained during routine meteorological soundings. In the troposphere, turbulence data have been provided by airline pilots and, occasionally, by research aircraft. On the other hand, the development of supersonic aircraft has raised the question of the turbulence hazard at altitudes above 40,000 ft. To investigate turbulence in the stratosphere (as well as at lower altitudes), it is desirable to consider all possible sources of information. In this paper rising balloons tracked by the highly accurate FPS-16 missile tracking radar are considered as a source of turbulence data. A system using this radar and special balloons has been developed for obtaining accurate and detailed wind measurements; pertinent papers have been given by Leviton (1962), Scoggins (1964), Murrow and Henry (1965), Reid (1965), MacCready (1965), Weinstein et al. (1966), and others.

The definition of turbulence that will be used herein is in the Eulerian sense, i.e., turbulence consists of erratic velocity variations in time at a particular point in space. In contrast, laminar flow (or "wind") at a point varies slowly with time even if large spatial variations (shears) exist over small distances. In turbulence analysis, assumptions are generally made relating different kinds of averages, particularly in time and space. A rising balloon gives only a single measurement at each altitude, and, therefore, does not provide perfectly un-

1 This work was performed under Contract AF 19(628)-5173 with the Air Force Cambridge Research Laboratories, Bedford, Mass. ambiguous measurements in either frame. However, where erratic variations in range occur as the balloon passes neighboring points with small delay, it is highly probable that turbulence rather than laminar flow is present. This hypothesis is basic to the present investigation.

\section{Radar response and self-induced balloon motions}

In order that turbulence may be deduced from the path of the rising balloon, it is necessary that position variations introduced by radar tracking and by selfinduced balloon motions be smaller than the eddy effects of the turbulent atmosphere. For reasons described later, we were particularly interested in the accuracy of slant-range measurements made by the FPS-16, and in the hunting characteristics of this equipment. A few plots of range measured at 0.1 -sec intervals showed smooth point-to-point changes, attesting to the high precision of this measurement. As to the hunting characteristics of the FPS-16, a discussion by Skolnik (1962) states that mechanical resonance frequencies are approximately $15 \mathrm{cps}$ and servo frequencies are $5 \mathrm{cps}$ or higher. Therefore, readings averaged over one-half second or longer should be relatively free from radar tracking errors.

The question of self-induced balloon motions has been treated in the references cited earlier. Large smooth balloons are in the supercritical regime of Reynolds number and experience appreciable self-induced aerodynamic motions due to eddy shedding. However, the Jimsphere and the one-meter Rose balloon have been found to be quite stable, and data from these are suitable for our purpose. The nature of the oscillations of 
Jimspheres as indicated in this study will be shown later, and these small regular motions can be distinguished from what appear to be random turbulent motions.

Very few three-dimensional measurements of free-air turbulence exist. Some recently available aircraft data (Jones and Atnip, 1964; Reiter and Burns, 1965) indicate approximate isotropy. Also, the measurements support the concept of the inertial subrange, i.e., the spectrum of eddy size is continuous from centimeters up to hundreds of meters, and the larger eddies contain higher eddy velocities. Therefore, a balloon rising at a speed of approximately $5 \mathrm{~m} \mathrm{sec}^{-1}$ will pass through a typical $50-\mathrm{m}$ turbulent eddy in about $10 \mathrm{sec}$, and has sufficient time to adapt to a large proportion of the eddy motion. Also, if gravity-inertia waves exist instead of turbulence, these should be detectable over similar distances as periodic (rather than random) oscillations.

\section{Method of data processing}

The basic FPS-16 data consist of slant range $R$, elevation angle $E$, and azimuth angle $A$ measured from the radar to the rising balloon at $0.1-\mathrm{sec}$ intervals. For the present preliminary investigation, data for ten Jimsphere flights were furnished to us by the Aero-Astrodynamics Laboratory, George C. Marshall Space Flight Center, NASA, Huntsville, Ala. The flights that we chose are Test Numbers 7975, 8959, 0356, 516-4, 0734, $1272-5,1654,2113,2579$, and 2581. Wind data for these flights have been presented by Scoggins and Susko (1965).

Normally, the first step in processing FPS-16 data has been to convert from $R, E, A$ to $x, y, z$ coordinates. For reasons given below, we believe that it is simpler to detect turbulence from the slant-range measurements than from $x, y, z$ coordinates or from the associated $u, v, w$ wind components. One factor is that the slant range is apparently the most accurate measurement of the FPS-16. Another reason for using slant range is that this quantity generally varies rather steadily with time (or altitude), and, in fact, is normally a monotonic increasing function. Where winds are weak, range increases slowly, and where they are strong it increases rapidly. However, large changes in speed are separated sufficiently in space that the range curve is quite smooth. At maxima or minima in wind speed profiles, the range profile will have an inflection point. Thus, the over-all trends of range versus time can be computed by simple, straight-forward techniques, whereas fitting a smooth curve to a wind profile is comparatively difficult and time-consuming. If the turbulence is isotropic, the eddy structure can be deduced as well from slant range (whatever its orientation) as from variations of the $x, y, z$ coordinates. As mentioned earlier, isotropy is generally supported by measurements and by theory. Nevertheless, it should be borne in mind that under conditions of strong winds, slant range tends to be large and elevation angle small. In turbulence associated with jet streams, slant-range variations will reflect primarily horizontal eddy components.

To determine eddy variations in slant range as a function of time (or altitude), one can first obtain a smooth curve of slant range versus time and then take deviations from this curve. We wish to use the smoothed curve in computing wind and its vertical derivatives, while the unsmoothed portion represents turbulence. Actually, the distinction between "wind" and "turbulence" is somewhat arbitrary. Generally, wind is computed over layers $1000 \mathrm{ft}$ thick or more, although smaller increments have been used with the FPS-16 data. The data processing that we chose is somewhat similar to procedures used by Scrase (1954) to estimate eddies from 1-min readings of upper winds. The computations are as follows. Ten values of range $R$ at 0.1 -sec intervals are averaged to give values at $1-\mathrm{sec}$ intervals; these are the basic data used in the analysis. This averaging tends to eliminate radar errors and reduces the number of points to an easily manageable size. Next a smoothed curve of $R$ is prepared as follows. A 1-min average of $R$ is computed and is considered to be centered at the midpoint in time. A linear interpolation is made between these 1-min averages to obtain the smoothed range $R_{s}$ at each second. Then, the deviations $\left(R-R_{s}\right)$ at each second may be plotted as functions of time or height, and since major trends have been removed, the departures indicate the small-scale motions that occurred. The rms value of $\left(R-R_{s}\right)$ in a given interval is a measure of the intensity of the turbulence in that portion of the sounding.

The values of azimuth and elevation angles are each treated in the same manner as range. Differential linear variations comparable to $\left(R-R_{s}\right)$ along two arcs orthogonal to $R$ can be computed (in spherical coordinates) as $R \times\left(E-E_{s}\right)$ and $(R \cos E) \times\left(A-A_{s}\right)$, respectively. We expect that these variations may be uscd to determine the degree of isotropy of the turbulence if elevation angles are sufficiently high. (At very low elevation angles, the angular deviations $\left(E-E_{s}\right)$ and $\left(A-A_{s}\right)$ appear to contain an undesirable level of noise. Further investigation of this aspect of the data is required.)

From the 1-min averaged values of $R, A$, and $E$, values of height above the spherical earth and of spherical arc length from the radar site to a point beneath the balloons are computed. By differencing these 1-min values and using appropriate trigonometric relations, the $u, v, w$ components of balloon motion are obtained over 1-min intervals. These were desired for correlation later on with the turbulence intensity in the same layers. In the computer program that performs these computations, features such as averaging times may be altered by simple modifications: 


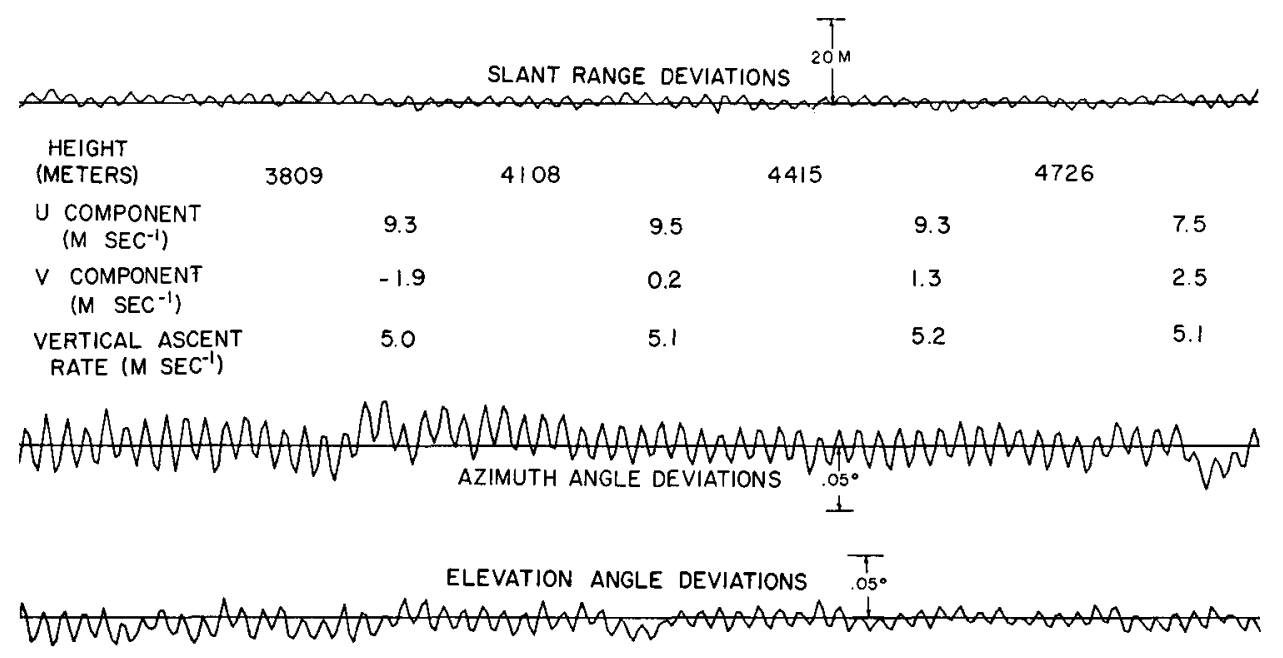

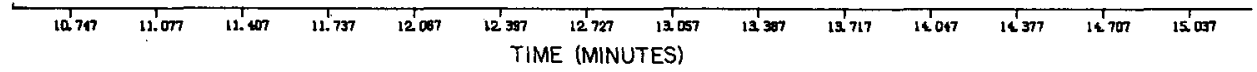

FIG. 1. Graphs of deviations of slant range, azimuth angle, and elevation angle from smoothed curves, and wind components over 1 -min intervals. Deviations show self-induced balloon oscillations. Data from Jimsphere flight 8959, 28 December 1964, near $4 \mathrm{~km}$ altitude.

\section{Results}

For each of the ten Jimsphere flights, the 1 -sec values of $\left(R-R_{s}\right),\left(A-A_{s}\right)$, and $\left(E-E_{s}\right)$ were plotted with a California Computer Company plotter as functions of time. For a flight $1 \mathrm{hr}$ or more in duration, the plotted record is about $10 \mathrm{ft}$ long and therefore cannot be reproduced here in its entirety. For the first $30 \mathrm{~min}$ or so of each flight (to altitudes of approximately $10 \mathrm{~km}$ ), the deviations show wave-like variations with a period of approximately $4 \mathrm{sec}$ and an amplitude in $\left(R-R_{s}\right)$ of several meters. These are evidently the small aero- dynamic motions of the Jimsphere, as noted by others. The azimuth deviations are approximately $90 \mathrm{deg}$ out of phase with the range deviations, while the elevation deviations are $180 \mathrm{deg}$ out of phase with the range. These relations are consistent with a helical path of the balloon.

Portions of machine-plotted graphs of range, azimuth and elevation deviations at 1 -sec intervals for Jimsphere flight 8959 are given in Figs. 1 and 2. [The plotted wind profile for this flight is Fig. 4 in the report of Scoggins and Susko (1965).] In Fig. 1, all three
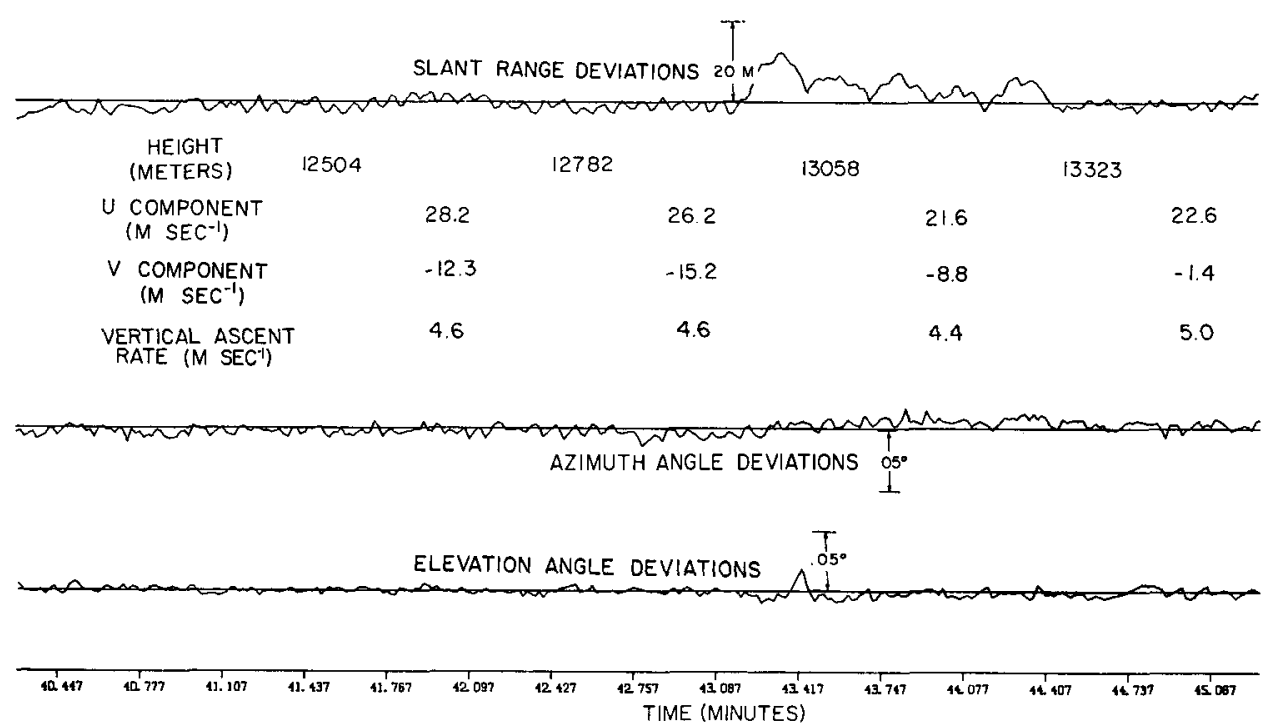

Fig. 2. Graphs of deviations of slant range, azimuth angle, and elevation angle from smoothed curves, and wind components over 1-min intervals. Range curve shows turbulent fluctuations Data from flight 8959 near $13 \mathrm{~km}$ altitude. 


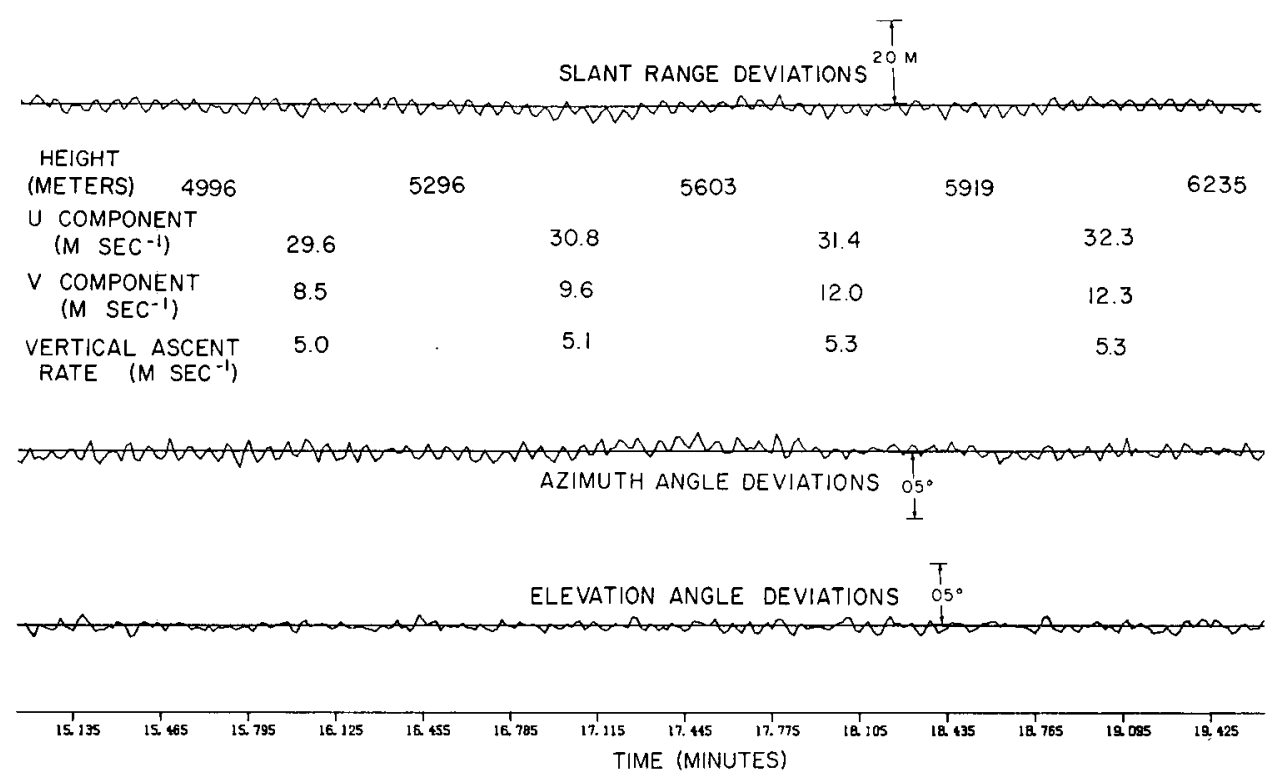

FIG. 3. Graphs of deviations of slant range, azimuth angle, and elevation angle from smoothed curves, and wind components over 1-min intervals. Deviations show self-induced balloon oscillations. Data from Jimsphere flight 516-4, 27 January 1965, near $5 \mathrm{~km}$ altitude.

curves exhibit consistent wave-like fluctuations as described above. The jaggedness of the plots is due partly to the plotter's interpolation between neighboring points but this feature does not affect our interpretation. In Fig. 2 at about $13,000 \mathrm{~m}$ altitude, the range curve has sudden large erratic oscillations. In our opinion, these indicate turbulence in a layer about $500 \mathrm{~m}$ thick. The wind data at 1-min intervals show shear that is consistent with turbulence. It is also interesting that a change in the balloon's ascent rate, probably due to changing vertical air motions, occurred in this same layer. At this time the elevation angle was approximately $18 \mathrm{deg}$ so that undue noise would not be expected.

Similar plots for Jimsphere flight 516-4 are given in Figs. 3, 4 and 5. Turbulent fluctuations appear to be present in the latter two figures. Approximate gust velocities may be calculated from the graphs as differences between range deviations, divided by the time difference. In Fig. 5, gusts determined in this way are

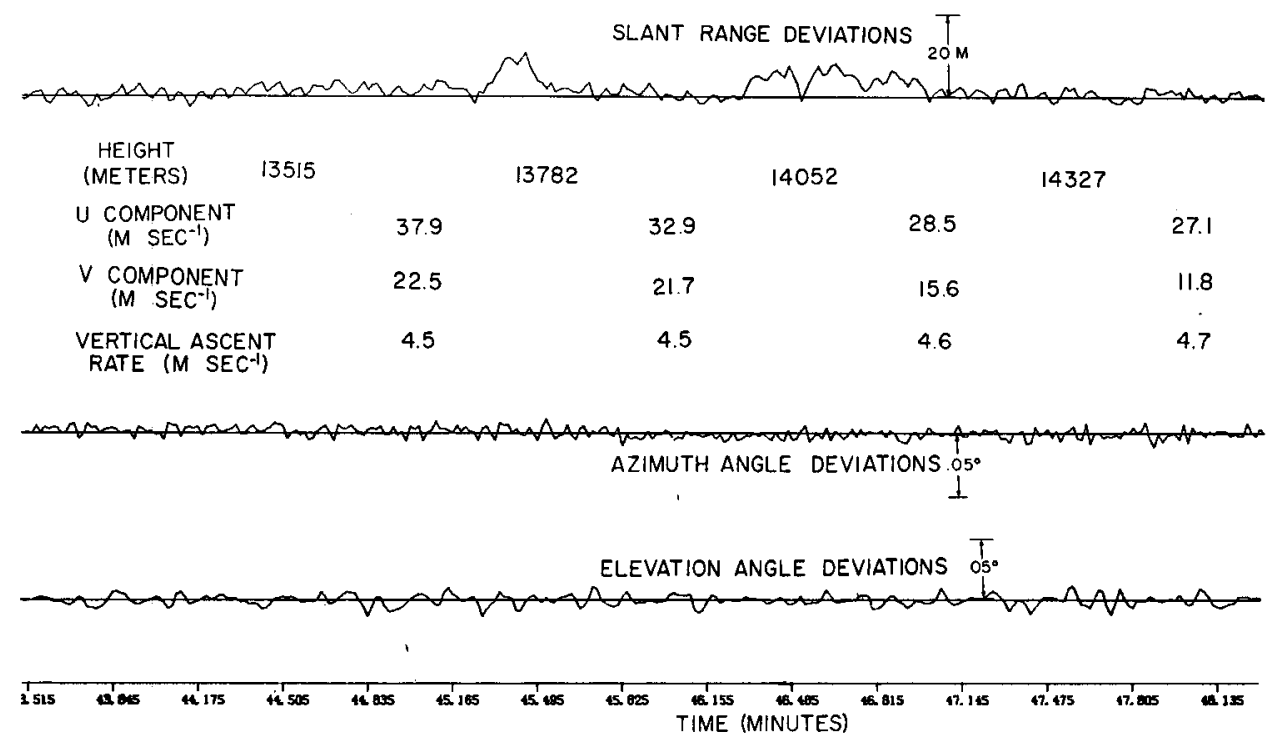

FIG. 4. Graphs of deviations of slant range, azimuth angle, and elevation angle from smoothed curves, and wind components over 1 -min intervals. Range curve shows turbulent fluctuations. Data from flight $516-4$ near $14 \mathrm{~km}$ altitude. 

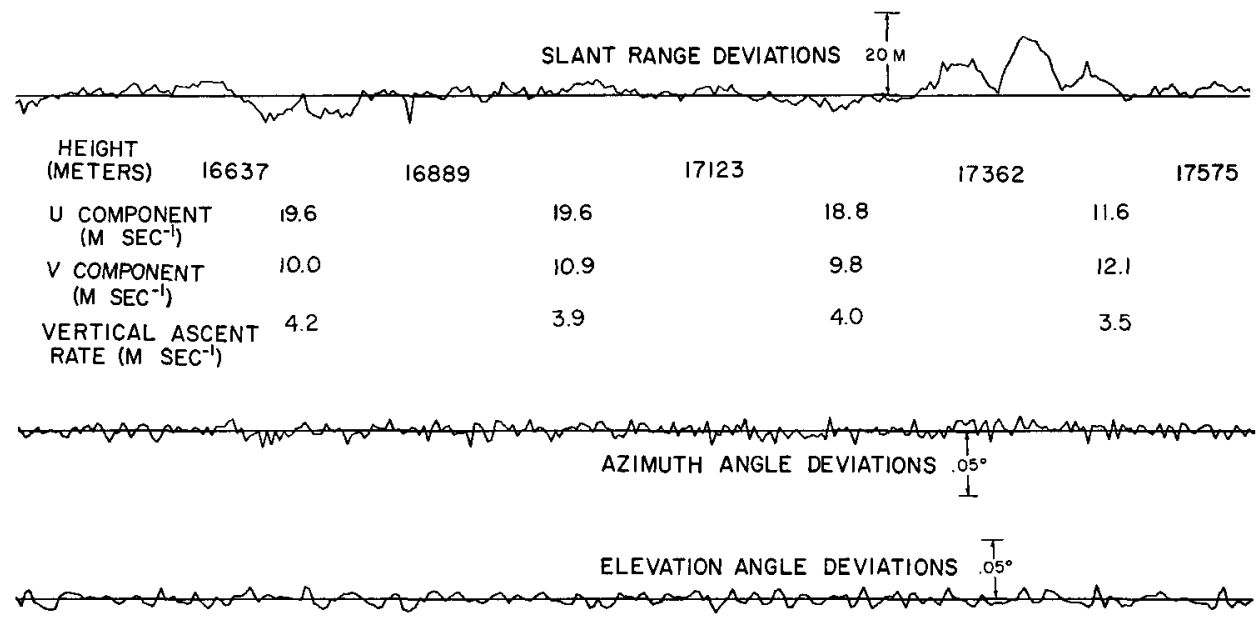

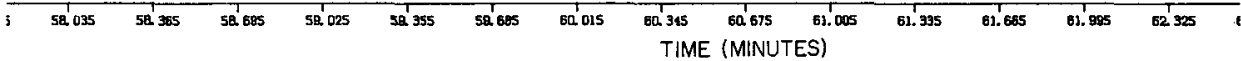

FIG. 5. Graphs of deviations of slant range, azimuth angle, and elevation angle from smoothed curves, and wind components over 1-min intervals. Turbulent fuctuations are approximately $3 \mathrm{~m} \mathrm{sec}^{-1}$, indicating light-to-moderate turbulence. Data from flight 516-4 near $17 \mathrm{~km}$ altitude.

approximately $3 \mathrm{~m} \mathrm{sec}^{-1}$, indicating turbulence on the threshold between light and moderate intensity. (At this time the elevation angle was $7.5 \mathrm{deg}$, still believed high enough to insure reliable data.) By processing data from additional flights, we expect to uncover cases of more highly developed turbulence, and to investigate the relationships between turbulence and the vertical wind profile.

\section{Summary}

The investigation of data from ten Jimsphere flights tracked by FPS-16 radar indicates that these data are an excellent source of information on turbulence. One major advantage of these observations is that the flights penetrate well into the stratosphere where other data are scarce. A second advantage is that winds and wind shear are obtained simultaneously so that turbulence intensity can be correlated with these quantities. A third factor is the relatively low expense of obtaining turbulence data. Disadvantages are the small number of FPS-16 radars available, and the fact that a rising balloon may pass through a turbulent layer rather quickly without obtaining a record long enough for a complete statistical analysis such as spectrum computations.

Acknowledgments. The writers are grateful to $\mathrm{Mr}$. Norman Sissenwine of the Air Force Cambridge Re- search Laboratories for the advice he provided during this study.

\section{REFERENCES}

Jones, J. W., and L. K. Atnip, 1964: High intensity gust investigation. Technical Report under Contract AF 33(600)-43501, Boeing Aircraft Co., Wichita, Kans., 79 pp.

Leviton, R., 1962: A detailed wind profile sounding technique. Air Force Surveys in Geophysics, No. 140, Air Force Cambridge Research Laboratories, Bedford, Mass., 187-195.

MacCready, P. B., 1965: Comparison of some balloon techniques. J. Appl. Meteor., 4, 504-508.

Murrow, H. N., and R. M. Henry, 1965: Self-induced balloon motions. J. Appl. Meteor., 5, 131-138.

Reid, D. F., 1965: Instability of spherical wind-sensing balloons. Air Force Surveys in Geophysics, No. 167, Air Force Cambridge Research Laboratories, Bedford, Mass., 213-227.

Reiter, E. R., and A. Burns, 1965: Atmospheric structure and clear-air turbulence. Atmospheric Science Technical Paper No. 65, Colorado State University, Ft. Collins, Colo., 17 pp.

Scoggins, J. R., 1964: Aerodynamics of spherical balloon wind sensors. J. Geophys. Res., 69, 591-598.

—, and M. Susko, 1965: FPS-16 radar Jimsphere wind data measured at the Eastern Test Range. NASA TM X-53290, G. C. Marshall Space Flight Center, Huntsville, Ala., 457 pp.

Scrase, F. J., 1954: Turbulence in the upper air, as shown by radar wind and radiosonde measurements. Quart. $J . R$. Meteor. Soc., 80, 369-376.

Skolnik, M. I., 1962: Introduction to Radar Systems. New York, McGraw-Hill Book Co., pp. 194-196.

Weinstein, A. I., E. R. Reiter and J. R. Scoggins, 1966: Mesoscale structure of $11-20 \mathrm{~km}$ winds. $J$. Appl. Meteor., 5, 49-57. 